\title{
Long-term urological follow-up of multicystic dysplastic kidneys: Is it still indicated in 2007 ?
}

\author{
Karen Psooy, MD, FRCSC, DABU
}

\begin{abstract}
Objective: To determine whether the current indications for the long-term urological follow-up of children with multicystic dysplastic kidneys (MCDKs) are supported by the literature.

Methods: The membership of the Pediatric Urologists of Canada was surveyed to determine if long-term urological follow-up was being performed, and if so, for what indications. A literature search using PubMed, EMBASE and a Conference Papers Index was performed to determine whether the indications listed were supported by the literature.

Results: A response rate of $72 \%$ was achieved for the survey, with most respondents following children with MCDK long-term. The main indications for longterm follow-up were the increased risk of Wilms' tumour (54\%) and hypertension $(32 \%)$, observation of the contralateral kidney $(43 \%)$ and involution of the MCDK (36\%). The literature search did not support long-term urological follow-up for any of these indications, provided unilateral MCDK was an isolated genitourinary abnormality. Although it is rare, a primary care physician could monitor for hypertension.
\end{abstract}

Conclusion: Long-term urological follow-up of children with "simple MCDK" is not supported by the literature, provided the diagnosis has been confirmed with a follow-up renal ultrasound at 12-24 months. Blood pressure monitoring by a primary care physician is recommended.

CUAJ 2007;1(2):113-6

\section{Introduction}

Prior to the advent of routine antenatal ultrasound in the 1980s, multicystic dysplastic kidneys (MCDKs) were considered rare and almost always presented with symptoms (i.e., urinary tract infections [UTIs], hypertension or pain) or as a palpable mass. Management at that time was a nephrectomy to eliminate the symptoms, to diagnose the mass, ${ }^{1}$ which at the time could not always be differentiated from a malignancy, ${ }^{2}$ or both. However, with antenatal ultrasound, MCDKs were identified in 1 of 4300 live births ${ }^{3}$ and could no longer be considered rare or to classically present with symptoms. ${ }^{1}$ With this change in epidemiology came a change in management, from nephrectomy to observation. ${ }^{1}$ In order to determine if this practice was safe, the Multicystic Kidney Registry was set up by the American Academy of Pediatrics Section of Urology in 1986. The purpose was to understand the prog- nosis of MCDKs in terms of involution and the risks of developing the previously associated complications. Preliminary data on this were published in 1993, stating that observation was safe and prophylactic nephrectomy did not seem justified. ${ }^{4}$ What remained unanswered by the data was how long patients should be observed and whether they needed to be observed at all. Unfortunately, no further updates from this registry have been published. Today, as these questions have remained unanswered after 20 years, some pediatric urologists are once again advocating nephrectomy, with the main indication being to avoid the cost, time and anxiety associated with long-term observation,, 5,6 wile others are introducing the idea of early discharge. ${ }^{7}$ I postulate that there is enough information in the literature to be able to answer the question of whether long-term urological observation is still indicated in children with MCDKs.

\section{Methods}

A survey was sent by email to the membership of the Pediatric Urologists of Canada (PUC), an affiliate of the Canadian Urological Association, in January 2006, asking if they followed children for MCDKs, and if so, for what indications (Box 1). A literature search using PubMed and EMBASE was then performed to assess the literature support for these indications. Key search terms included "multicystic kidney" and "multicystic dysplastic kidney," paired with "Wilms' tumor," "hypertension" and "natural history." The titles of related articles were reviewed on all key articles. Articles were read if the titles and abstracts were pertinent to the question to be answered by the review. A Conference Papers Index was also searched for case presentations of Wilms' tumour. 


\section{Results and Discussion}

Of the 39 PUC members, 28 responded. This resulted in a $72 \%$ response rate. Only 1 responding urologist $(3 \%)$ offered nephrectomy, with the main indications being to avoid long-term follow-up and to avoid a more difficult nephrectomy at an older age. There was only 1 urologist (3\%) who did not follow children with MCDKs, as at their centre, the pediatric nephrologists closely follow these children. Three urologists (11\%) followed patients with MCDKs for 2 years or less, and the remainder followed with appointments and renal ultrasounds. While the survey did not define "follow" as long-term, based on a review of the PUC survey results, it is apparent that the question was appropriately interpreted to mean follow-up beyond 1 or 2 years. The main indications for long-term follow-up were the risk of developing ipsilateral Wilms' tumour (according to $54 \%$ of respondents), observation of the contralateral kidney (according to $46 \%$ of respondents), to confirm involution (as opposed to growth) (36\% of respondents) and to observe possible hypertension (according to $32 \%$ of respondents). The result of the literature review, which focused on each of these main indications, follows.

A larger, international survey of pediatric urologists might have identified additional indications

\footnotetext{
Box 1. Multicystic dysplastic kidney (MCDK) survey sent to the membership of the Pediatric Urologists of Canada

1. Do you routinely offer prophylactic nephrectomy for MCDKs?

a. If so, what is (are) your MAIN indication(s)

i. Risk of Wilms' Tumour

ii. Risk of hypertension

iii. Avoidance of long term follow-up

iv. Other

2. Do you routinely follow MCDKs with ultrasounds

and appointments?

a. If so, what is (are) your MAIN indication(s):

i. Risk of Wilms' Tumour

ii. Risk of hypertension

iii. Observation of contralateral kidney

iv. Other

3. If you were convincingly shown that neither of these treatment plans were justified, based on the literature, would you support the development of a Canadian Urological Association guideline that states that early discharge is an acceptable option?
}

for follow-up. However, review of the international literature on MCDK did not suggest anything beyond the historical indications.

\section{Risk of Wilms' tumour}

Of survey respondents, 54\% considered the risk of developing Wilms' tumour one of the main indications for follow-up.

The literature suggests that between 1983 and 1998, only 8 cases of Wilms' tumour development in a MCDK were reported in the United States. ${ }^{8-10}$ In an editorial comment, Beckwith questioned the diagnosis of 3 of these cases, ${ }^{9}$ leaving only 5 US cases. Three cases from outside the United States have also been reported..$^{10}$ Of all these cases, only 1 documents the diagnosis of a Wilms' tumour by follow-up renal ultrasound. ${ }^{11}$ The literature search found no additional cases of Wilms' tumour in MCDKs since 1998. The limited data available on these cases does not permit the identification of any high-risk features predictive of Wilms' tumour development.

While it seems very reassuring that no cases of Wilms' tumour in MCDKs have been published since 1998, one cannot assume that they have ceased to occur. Over the years it has become more difficult to publish case reports in major medical journals. In an effort to overcome this potential bias, a Conference Papers Index was searched; however, no cases were found.

There have been efforts to estimate the risk of Wilms' tumour developing in an MCDK, based on the number of US cases reported between 1983 and 1997. This has resulted in a risk estimate of somewhere between $0.03 \%$ and $0.1 \%$. In an effort to determine the probability of Wilms' tumour in an MCDK, Narchi' ${ }^{12}$ performed a systematic review of all published cohort studies of MCDKs and identified 26 papers from 1986 to 2004, for a total of 1041 MCDKs that were followed conservatively. Wilms' tumour was not identified in any of these cases. Within these series, an additional 178 children had nephrectomies, but none owing to malignancy. Narchi concluded that the probability of Wilms' tumour developing in MCDKs is nil. The available literature has caused others to conclude that there is no evidence to suggest MCDK patients are at an increased risk of developing Wilms' tumour. ${ }^{13}$ 


\section{Observation of the contralateral kidney}

Of survey respondents, $43 \%$ considered observing the contralateral kidney as one of the main indications for follow-up. Feldenberg and Siegel followed 35 cases of MCDK to determine the probability of UTI, chronic renal failure and end stage renal disease. ${ }^{14}$ They subclassified the cases into "simple MCDK" (defined by unilateral dysplasia with a normal contralateral kidney with compensatory hypertrophy and no associated genitourinary anomalies on renal ultrasound or physical examination) and "complex MCDK" (defined as bilateral dysplasia, or abnormalities of the contralateral kidney or genitourinary tract detected by renal ultrasound or physical examination). After 5 years of follow-up, only 1/14 cases of "simple MCDK" developed a UTI and none developed chronic renal failure or end stage renal disease. Conversely, after 8 years of follow-up, of the 21 cases of "complex MCDK," $29 \%$ developed UTI, 29\% developed chronic renal failure and an additional $21 \%$ went on to develop end stage renal disease.

This suggests that close urological follow-up of the contralateral kidney in "simple MCDK" is not indicated, but those with "complex MCDK" should be followed by a urologist and nephrologist or both, depending on the associated condition. Confirmation of "simple MCDK" requires confirmation of compensatory hypertrophy, which requires at least 1 additional renal ultrasound at age 12-24 months, before patients are discharged from follow-up.

A discussion regarding whether nephrological follow-up is needed and what such follow-up might entail in children with "simple MCDK" is beyond the scope of this paper. Children with a normal solitary functioning kidney owing to unilateral agenesis or unilateral nephrectomy have a small increased risk for proteinuria and renal insufficien$\mathrm{cy}^{15,16}$ and there is local variation in terms of whether these children ever see a nephrologist. However, if such a child were to be followed by a nephrologist, it should be follow-up designed for the solitary functioning kidney, not for the MCDK, and thus would not be in place of urological follow-up.

\section{Confirmation of involution}

When asked for "other" reasons for follow-up, 36\% of respondents said that they would perform follow-up to confirm either involution, resolution or lack of growth in the MCDK. Additional comments suggest that the combined reasons for this include confirming the diagnosis of MCDK and ruling out development of tumour. While it is essential to be satisfied with the diagnosis of MCDK before a patient is discharged, the MCDK Registry observed growth and lack of involution at up to 5 years, ${ }^{4}$ which is not in contrast with the diagnosis. The ultrasonographic criteria to diagnose classic MCDKs are clear, making a misdiagnosis of cystic malignancy unlikely when these criteria are identified by an ultrasonographer experienced in pediatrics ${ }^{17}$; difficulties differentiating hydronephrotic MCDK from severe ureteropelvic junction obstruction are more likely. ${ }^{18}$ In indeterminate cases, a renal scan showing no perfusion is supportive of an MCDK diagnosis, but evidence of some function does not necessarily rule it out. ${ }^{19}$

The risk of tumour development is discussed in a previous section.

\section{Risk of hypertension}

Thirty-two percent of survey respondents considered the risk of hypertension as an indication for follow-up. Based on case reports, there is no doubt that hypertension can be associated with MCDKs. There are cases where nephrectomy of an MCDK has resulted in cure of hypertension, even in 1 case where the MCDK was no longer seen on renal ultrasound..$^{20}$ Other case reports have shown that even with a history of a remote prophylactic nephrectomy, hypertension can develop. This suggests that the etiology may be owing to abnormalities in the contralateral kidney. ${ }^{21}$ In an effort to determine if the risk of hypertension in children with MCDKs was actually higher than that of the general population, Narchi analyzed all the published MCDK cohort studies reporting on hypertension. ${ }^{22}$ Of the 1115 children observed in 29 studies and followed for a mean of 3.5 years, 6 children developed hypertension. Using aggregation of the data, the probability of developing hypertension in MCDKs was therefore calculated at $0.54 \%$, compared with the risk of the general population of $1 \%-4.5 \%$. Narchi concluded that the risk of developing hypertension is no higher in MCDK patients than it is in the general pediatric population.

Although hypertension can certainly develop 
in a child with a history of MCDK, the risk does not appear to be more than that of the general pediatric population. Routine blood pressure monitoring is probably indicated in this population; however, it can be done by a primary care physician. Hypertension does not require a urologist's involvement, unless the MCDK is considered a likely etiology, in which case, nephrectomy should be considered.

\section{Conclusion}

After 20 years of observation, the literature has not only shown that observation is safe, but it also supports Onal and Kogan's conclusion that early discharge from urological follow-up is safe.

The indications that Canadian pediatric urologists use for long-term follow-up of MCDKs are not supported by the literature:

- The increased risk of developing Wilms' tumor appears negligible, if not nonexistent.

- The contralateral kidney should be observed by a urologist if a urological abnormality warranting observation is identified. However, if the child has a "simple MCDK" with confirmed compensatory hypertrophy, there does not appear to be a need for ongoing urological follow-up.

- Involution is not required to confirm a benign course for MCDKs and thus the observation of involution is not necessary. Satisfaction of the correct diagnosis of MCDKs is required for early discharge and can be done with ultrasound criteria identified by those experienced in pediatrics, and with nuclear renography, if necessary.

- The risk of hypertension is low, but it has been observed in children with MCDKs, regardless of whether their MCDK has been removed. Blood pressure monitoring by a primary care physician is recommended.

This current review of the literature suggests that a urologist's role in the care of a child with MCDKs could be safely changed. Rather than being a longterm observer, the urologist could be one who ensures the diagnosis is correct, rules out associated abnormalities that warrant follow-up by a urologist or a nephrologist, confirms "simple MCDKs" with an ultrasound at age 12-24 months and recommends blood pressure monitoring by the primary care physician.
The Division of Pediatric Urology, Winnipeg Children's Hospital, Winnipeg, Man.

This article has been peer reviewed.

Competing interests: None declared.

\section{References}

1. Welch TR, Wacksman J. The changing approach to multicystic dysplastic kidney in children. J Pediatr 2005;146:723-5.

2. Karmazyn B, Zerin JM. Lower urinary tract abnormalities in children with multicystic dysplastic kidney. Radiology 1997:203:223-6.

3. Gordon AC, Thomas DFM, Arthur RJ, et al. Multicystic dysplastic kidney: is nephrectomy still appropriate? J Urol 1988;140:1231-4.

4. Wacksman J, Phipps L. Report of the multicystic kidney registry: preliminary findings. J Urol 1993; 150:1870-2.

5. Steven $L C$, Li AGK, Driver CP, et al. Laparoscopic nephrectomy for unilateral multicystic dysplastic kidney in children. Surg Endosc 2005;19:1135-8.

6. Kaneko K, Yamashiro Y, Yamataka A, et al. Nephrectomy for multicystic dysplastic kidneys: a new therapeutic option. Pediatr Nephrol 2005;20:690-1.

7. Onal B, Kogan BA. Natural history of patients with multicystic dysplastic kidney what followup is needed? J Urol 2006;176:1607-11.

8. Homsy YL, Anderson JH, Oudihane K, et al. Wilms tumor and multicystic dysplastic kidney disease. J Urol 1997;158:2256-9.

9. Beckwith JB. Editorial comment. J Urol 1997;158:2259-60.

10. Perez $\mathrm{LM}$, Naidu SI, Joseph DB. Outcome and cost analysis of operative versus nonoperafive management of neonatal multicystic dysplastic kidneys. J Urol 1998;160:1207-11.

11. Oddone M, Marino C, Sergi C, et al. Wilms' tumor arising in a multicystic kidney. Pediatr Radiol 1994;24:236-8.

12. Narchi H. Risk of Wilms' tumour with multicystic kidney disease: a systematic review. Arch Dis Child 2005;90:147-9.

13. Truong LD, Choi YJ, Shen SS, et al. Renal cystic neoplasms and renal neoplasms associated with cystic renal diseases: pathogenetic and molecular links. Adv Anat Pathol 2003; 10:135-59.

14. Feldenberg LR, Siegel NJ. Clinical course and outcome for children with multicystic dysplastic kidneys. Pediatr Nephrol 2000; 14:1098-101.

15. Argueso LR, Ritchey ML, Boyle ET Jr, et al. Prognosis of patients with unilateral renal agenesis. Pediatr Nephrol 1992;6:412-6.

16. Argueso LR, Ritchey ML, Boyle ET Jr, et al. Prognosis of children with solitary kidney after unilateral nephrectomy. J Urol 1992; 148:747-51.

17. Stuck KJ, Koff SA, Silver TM. Ultrasonic features of multicystic dysplastic kidney: expanded diagnostic criteria. Radiology 1982;143:217-21.

18. Sanders RC, Hartman DS. The sonographic distinction between neonatal multicystic kidney and hydronephrosis. Radiology 1984;151:621-5.

19. Metcalfe PD, Wright JR Jr, Anderson PAM. MCDK not excluded by virtue of function on renal scan. Can I Urol 2002;9:1690-3.

20. Webb NJA, Lewis MA, Bruce J, et al. Unilateral multicystic dysplastic kidney: the case for nephrectomy. Arch Dis Child 1997;76:31-4.

21. Kuwertz-Broeking E, Brinkmann OA, VonLengerke H-J, et al. Unilateral multicystic dysplastic kidney: experience in children. BJU Int 2004;93:388-92.

22. Narchi H. Risk of hypertension with multicystic kidney disease: a systematic review. Arch Dis Child 2005:90:921-4.

Correspondence: Dr. Karen Psooy, Division of Pediatric Urology, Winnipeg Children's Hospital, HSC, FE 011-840 Sherbrook St., Winnipeg MB R3A 1S1; kpsooy@ exchange.hsc.mb.ca 